\title{
ANÁLISIS DEL CONTENIDO POLÍNICO DE MIELES PRODUCIDAS POR APIS MELLIFERA L. (HYMENOPTERA: APIDAE) EN EL ESTADO DE TABASCO, MÉXICO
}

\author{
Blanca Patricia Castellanos-Potenciano, ${ }^{1}$ Elia RAMíreZ- \\ ARRIAGA ${ }^{2}$ \& JUAN MANUEl ZALDIVAR-CRUZ ${ }^{3}$ \\ ${ }^{1,3}$ Programa de Maestría en Ciencias en Producción Agroalimentaria en el Trópico. Colegio de \\ Postgraduados, Campus Tabasco, Km. 3.5 Periférico Carlos A. Molina s/n. Carretera Cárdenas- \\ Huimanguillo.86500, Cárdenas, Tabasco. ${ }^{1}<$ bcastellanos@colpos.mx $>$; ${ }^{3}<$ zaldivar@colpos.mx>. \\ ${ }^{2}$ Laboratorio de Palinología, Instituto de Geología, Universidad Nacional Autónoma de México \\ (UNAM), Coyoacán, 04510, México, Distrito Federal. ${ }^{2}<$ elia@servidor.unam.mx>.
}

Castellanos-Potenciano, B. P., E. Ramírez-Arriaga \& J. M. Zaldivar-Cruz. 2012. Análisis del contenido polínico de mieles producidas por Apis mellifera L. (Hymenoptera: Apidae) en el estado de Tabasco, México. Acta Zoológica Mexicana (n. s.), 28(1): 13-36.

RESUMEN. Se estudiaron 40 muestras de miel de Apis mellifera L. con métodos melisopalinológicos, correspondientes a cuatro subregiones del estado de Tabasco. El polen de 29 taxa, la mayoría perteneciente a la flora nativa, fueron importantes $(\geq 10 \%)$ : Avicennia germinans (Acanthaceae); Borreria verticillata (Rubiaceae); Bursera simaruba (Burseraceae); Cecropia obtusifolia (Moraceae); Coccoloba aff. diversifolia (Polygonaceae), Conocarpus sp. (Combretaceae), Rumex sp. 1. (Polygonaceae), Eleocharis sp. 1 (Cyperaceae); Eragrostis sp. (Poaceae), Asteraceae sp. 1 y sp. 2. (Asteraceae), Andira sp. (Fabaceae), Diphysa carthagenensis (Fabaceae), Erythrina sp. 1(Fabaceae), Haematoxylum campechianum (Fabaceae), Heliocarpus appendiculatus (Tiliaceae), Machaerium sp. (Fabaceae); Mimosa albida (Fabaceae); Mimosa pigra var. berlandieri (Fabaceae); Phyla nodiflora (Verbenaceae); Piper sp. 1, sp. 2 y sp. 3. (Piperaceae), Quercus oleoides (Fagaceae); Spondias mombin (Anacardiaceae); Spondias radlkoferi (Anacardiaceae); Cocos nucifera (Arecaceae), Muntingia calabura (Elaeocarpaceae) y Zea mays (Poaceae). En general, se caracterizaron 14 mieles como monoflorales, 7 biflorales y 19 multiflorales. El mayor número de mieles fue del grupo II, conteniendo de 20,000 a 100,000 granos de polen en diez gramos de miel. Con base a los parámetros ecológicos, la explotación de recursos por A. mellifera fue más homogénea cuando se presentó una mayor diversidad de especies botánicas y un comportamiento de recolecta heterogéneo que coincidió con índices de diversidad bajos. Se encontró correlación entre algunas subregiones por la presencia de Mimosa albida, Bursera simaruba y Cecropia obtusifolia.

Palabras clave: caracterización de miel, Apis mellifera, melisopalinología, origen botánico.

Castellanos-Potenciano, B. P., E. Ramírez-Arriaga \& J. M. Zaldivar-Cruz. 2012. Analysis of honey pollen content produced by Apis mellifera L. (Hymenoptera: Apidae) at Tabasco State, Mexico. Acta Zoológica Mexicana (n. s.), 28(1): 13-36.

Recibido: 01/06/2010; aceptado: 10/10/2011 
ABSTRACT. We studied 40 honey samples from Tabasco State, Mexico with melissopalynology methods. Pollen grains of 29 taxa were important ( $\geq 10 \%)$ : Avicennia germinans (Acanthaceae); Borreria verticillata (Rubiaceae); Bursera simaruba (Burseraceae); Cecropia obtusifolia (Moraceae); Coccoloba aff. diversifolia (Polygonaceae), Conocarpus sp. (Combretaceae), Rumex sp. 1(Polygonaceae), Eleocharis sp. 1 (Cyperaceae); Eragrostis sp. (Poaceae), Asteraceae sp. 1 y sp. 2. (Asteraceae), Andira (Fabaceae), Diphysa carthagenensis (Fabaceae), Erythrina (Fabaceae), Haematoxylum campechianum (Fabaceae), Heliocarpus appendiculatus (Tiliaceae), Machaerium sp. (Fabaceae); Mimosa albida (Fabaceae); Mimosa pigra var.berlandieri (Fabaceae); Phyla nodiflora (Verbenaceae); Piper sp. 1, sp. 2 y sp. 3.(Piperaceae), Quercus oleoides (Fagaceae); Spondias mombin (Anacardiaceae); Spondias radlkoferi (Anacardiaceae); Cocos nucifera (Arecaceae), Muntingia calabura (Elaeocarpaceae) у Zea mays (Poaceae). In general, 14 monofloral, 7 bifloral and 19 multifloral honey samples were characterized. Most samples contained from 20,000 to 100,000 pollen grains per ten grams of honey. According to ecological parameters, homogeneous resource exploitation was registered with high diversity indexes and heterogeneous forage behavior matched with low H' values. We registered significant correlationships between some localities by the presence of Mimosa albida, Bursera simaruba and Cecropia obtusifolia.

Key words: Honey characterization, Apis mellifera, melissopalynology, botanic origin.

\section{INTRODUCCIÓN}

El néctar es una sustancia acuosa, rica en azúcares, aminoácidos, minerales y sustancias aromáticas, producido por las plantas y éstas lo ofrecen como principal recompensa a los polinizadores (Loayza \& Ríos 1999). Las abejas son atraídas por el néctar y durante su recolección realizan el transporte de polen de las anteras al estigma en un gran número de especies de angiospermas, por lo que son consideradas excelentes polinizadores en diversos ecosistemas (Simó 2002). Estos himenópteros muestran preferencias temporales por determinados recursos florales, los cuales son conocidos como flora melífera o flora apícola (Müller 1996, Price 1997).

En México, la mayor parte del conocimiento de la flora apícola se basa en observaciones de campo y entrevistas con apicultores, lo cual sobreestima el valor real de la flora melífera dado el carácter subjetivo de cada observador. Entre los estudios apícolas que han sido realizados con este método se encuentran los de Ayala (2001), quien reportó 38 especies de la flora melífera en la Península de Yucatán y Porter (2001) que citó más de 100 especies melíferas en el período de un año en la zona de La Montaña, en el estado de Campeche.

Por otro lado, la Secretaría de Agricultura, Ganadería, Desarrollo Rural, Pesca y Alimentación de México (SAGARPA) ha publicado catálogos de "Flora Nectarífera y Polinífera" de varios estados de la República como Michoacán, Chiapas, Guerrero, Veracruz y Tabasco (SAGARPA 1999, 2002, 2003, 2004) y regiones como la Península de Yucatán (SAGARPA 1998). Para conocer el origen botánico de las mieles de A. mellifera no son suficientes las observaciones de campo, ya que dificulta mucho la validación de que una miel pueda provenir de una especie u otra, o bien de algún 
cultivo en específico, simplemente porque estos se encuentren próximos al apiario (Martínez-Hernández\& Ramírez-Arriaga 1997).

Entre los estudios melisopalinológicos realizados en México, se cuenta con los de Ramírez-Arriaga (1989), quien analizó los recursos explotados por Plebeia sp. en el Soconusco, Chiapas en dos zonas diferentes, reportando 22 y 26 especies de importancia para cada una de las zonas; Acosta-Castellanos \& Palacios Chávez (2001) determinaron las plantas de interés apícola en la zona de Pluma Hidalgo, Oaxaca a partir de dos muestras de miel y cargas de polen de dos apiarios durante un ciclo anual; Villanueva (2002), identificó las plantas poliníferas y estrategias de pecoreo de A. mellifera L. en la península de Yucatán; Piedras \& Quiroz (2007) citaron cuatro especies como importantes en dos mieles de la porción Sur del Valle de México; Ramírez-Arriaga \& Martínez-Hernández (2007) mencionan nueve especies botánicas de importancia, encontraron conducta heterogénea y correlación en los hábitos de pecoreo de A. mellifera L. y Scaptotrigona mexicana Guérin en la Sierra Norte de Puebla y Quiroz \& Arreguín (2008) señalan siete especies melíferas pertenecientes a cuatro familias en el estado de Morelos. Villanueva-Gutiérrez et al. (2009) reportan 16 taxas en mieles monoflorales de importancia en la península de Yucatán y Ramírez-Arriaga et al. (2011) recuperaron 64 taxa con porcentajes mayores al 1\% de mieles colectadas en la costa de Oaxaca, mismas que fueron caracterizadas como monoflorales, biflorales, oligoflorales y poliflorales.

Por otro lado, en los trabajos apícolas realizados mediante observaciones de campo, el número de especies de la flora melífera es extenso, hasta 100 especies o más. En contraste, investigaciones melisopalinológicas han podido constatar, que sólo un número reducido, hasta 26 especies, llegan a ser importantes en cada región.

En el estado de Tabasco existe una amplia diversidad botánica con potencial apícola, la cual ha sido muy poco estudiada, ya que solamente se ha reportado un trabajo sobre la caracterización del ciclo apícola y flora nectarífera y polinífera en la zona de la Chontalpa (Cárdenas 1985) y la publicación de la "Flora nectarífera y polinífera del estado de Tabasco" (SAGARPA 2004), mientras que la mayoría de las investigaciones se han centrado en las propiedades fisicoquímicas y el perfil de aromas de mieles maduras (Viuda-Martos et al. 2010), la actividad antioxidante de las mieles cosechadas (Ruiz-Navajas et al. 2011) y en la caracterización melisopalinológica y fisicoquímica de las mieles maduras en dos temporadas de cosecha con la finalidad de obtener su denominación de origen geográfico (Córdova et al. 2009). En síntesis, poco es conocido sobre las especies melíferas nativas y cultivadas en el estado de Tabasco con base en la caracterización polínica de miel de A. mellifera, por lo que en el presente estudio se determinaron los principales recursos botánicos explotados por la abeja común a través del análisis palinológico de mieles colectadas en seis municipios pertenecientes a cuatro subregiones del estado de Tabasco y se aportó conocimiento sobre el papel ecológico de $A$. mellifera. 


\section{MATERIALES Y MÉTODOS}

Los muestreos se llevaron a cabo durante el periodo Febrero-Mayo de 2009, colectándose un total de 40 muestras de miel en apiarios ubicados en cuatro subregiones del estado de Tabasco: a) en la subregión Centro se colectaron ocho muestras del municipio Centro (92 $53^{\circ} 58.92^{\prime \prime}$; $\left.18^{\circ} 3^{\prime} 33.26^{\prime \prime}\right)$; b) en la subregión de la Chontalpa se colectaron ocho muestras, tres del municipio de Cárdenas (9335'0.14'”; $17^{\circ} 59^{\prime} 5.82^{\prime \prime}$ ) y cinco en el municipio de Paraíso (93 ${ }^{\circ} 14^{\prime} 25.28^{\prime}$ '; $18^{\circ} 25^{\prime} 57.04$ ' '); c) en la subregión Ríos se recabaron 16 muestras, ocho en el municipio de Balancán (91³2’30.02',; $17^{\circ} 49^{\prime} 54.12^{\prime \prime}$ ) y ocho del municipio de Tenosique (92 $19^{\circ} 53.87^{\prime \prime} ; 17^{\circ} 27^{\prime} 16.5^{\prime \prime}$ ), por último, d) en la subregión de la Sierra se colectaron ocho muestras de miel en el municipio Tacotalpa (9241'11.67'’; 17²6'5.1'”) (Fig. 1).

Toma de muestras de miel. La toma de muestras se realizó cada 15 días, recolectándose $100 \mathrm{ml}$ de miel en cada apiario con la ayuda de una pipeta de $5 \mathrm{ml}$. Cada muestra

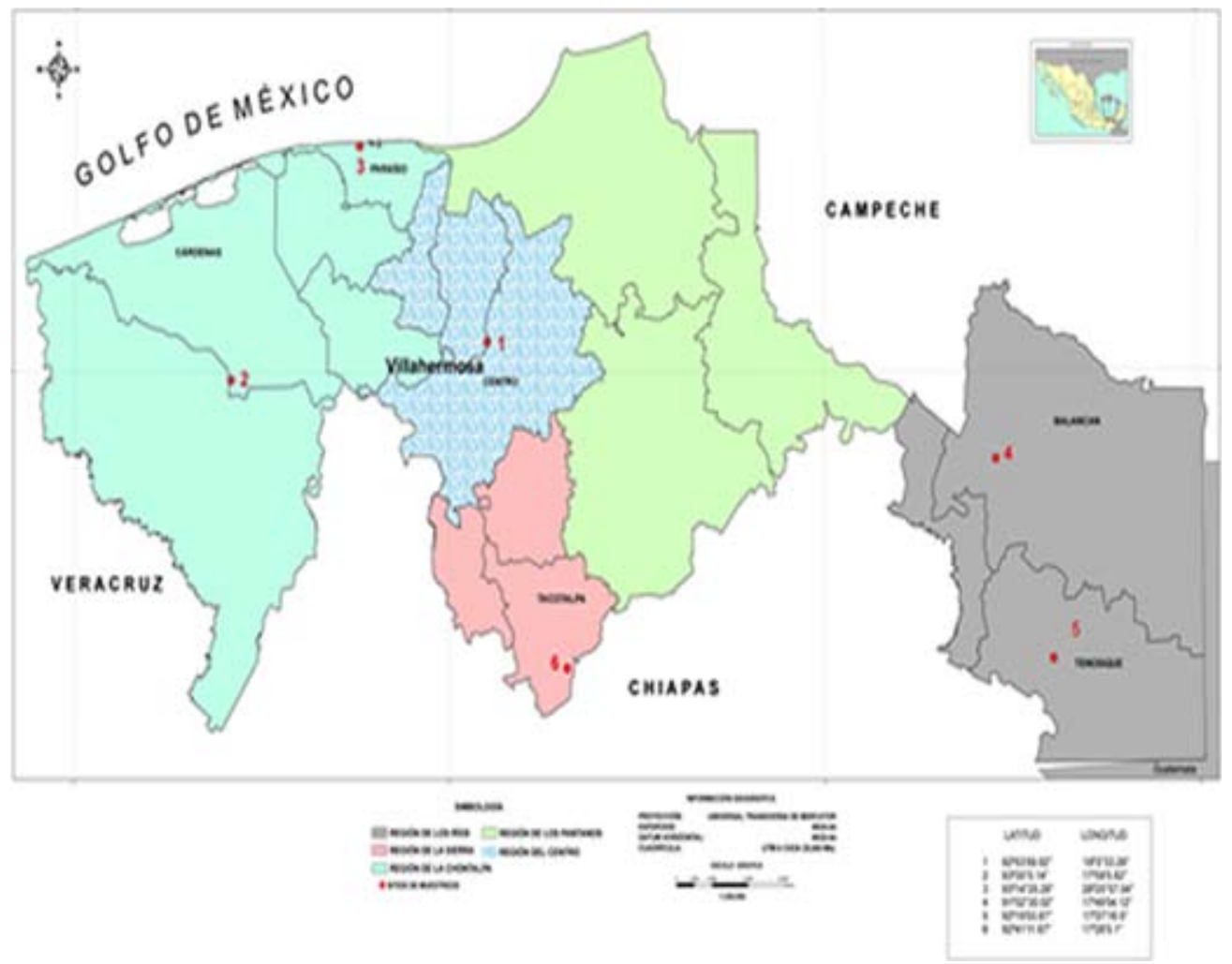

Figura 1. Ubicación de las subregiones del estado de Tabasco y municipios muestreados. 
de miel fue almacenada en tubos independientes Falcon de $50 \mathrm{ml}$, etiquetados con el nombre del municipio, número y fecha de colecta.

Preparación de las muestras. Todas las muestras fueron procesadas y analizadas en el Laboratorio de Palinología del Instituto de Geología de la UNAM (IGLUNAM). Con la finalidad de tener una mejor representatividad del contenido polínico de las muestras de miel, se modificó la técnica convencional de Louveaux et al. (1978), quintuplicando la cantidad de miel procesada. En este sentido, $50 \mathrm{ml}$ de miel por muestra se diluyeron en $100 \mathrm{ml}$ de agua destilada. Los granos de polen contenidos en la miel fueron concentrados por centrifugación, se agregó una pastilla de esporas marcadoras de Lycopodium clavatum por muestra, luego se procedió a la acetólisis siguiendo el método de Erdtman (1969). Posteriormente, se elaboraron 4 laminillas permanentes con gelatina glicerinada por muestra, las cuales fueron registradas e incorporadas a la colección palinológica del IGLUNAM.

Análisis melisopalinológicos de las muestras. Los análisis consistieron en la descripción e identificación de los granos de polen empleando un microscopio óptico Carl-Zeiss y el objetivo 100X. La identificación de los granos de polen a diferentes niveles taxonómicos se realizó por comparación, con la ayuda de claves polínicas, de la Colección de Referencia del Laboratorio de Palinología del IGLUNAM y utilizando artículos científicos especializados. Los tipos polínicos más importantes fueron fotografiados con un fotomicroscopio Zeiss Axiolab con cámara digital AxioCam ICc1.

Análisis cuantitativo del polen. Los porcentajes de cada tipo polínico se calcularon a partir del conteo de 500 granos de polen por muestra, en transectos al azar (Louveaux et al. 1978) y con el objetivo 100X. Adicionalmente, se contaron las esporas de Lycopodium clavatum L. para obtener la cantidad absoluta de granos de polen por gramo de miel (Stockmarr 1971). Especies con porcentajes mayores o iguales al 10\% fueron consideradas como importantes.

Para calcular la cantidad absoluta de los granos de polen por gramo de muestra se utilizó la siguiente fórmula, de acuerdo a Stockmarr (1971) y Maher (1981):

$$
\text { Pconc }=\boldsymbol{R M} / \mathrm{V}
$$

Donde:

$\boldsymbol{P} \boldsymbol{c o n c}$ es el polen por gramo; $\boldsymbol{R}$ son los granos de polen contados/el número de esporas contadas; $\boldsymbol{M}$ es el número de esporas adicionadas en cada tableta; $\boldsymbol{V}$ es el peso total de la muestra de miel. Las concentraciones de polen fueron clasificados siguiendo el esquema de Maurizio (1939). 
Una vez obtenida la cantidad absoluta de granos de polen por gramo de miel, el polen de los taxa se clasificó como predominante $(\mathrm{P} \geq 45 \%)$, secundario $(\mathrm{S}=16-45 \%)$ de menor importancia $(\mathrm{I}=3-15 \%)$ o polen traza $(\mathrm{M} \leq 3 \%)$ (Louveaux et al.1978).

Caracterización botánica de las mieles. Por otro lado, las mieles fueron caracterizadas como "monoflorales" cuando en su composición presentó una especie con porcentaje de polen superior o igual a $45 \%$ y "multiflorales, mixta o polifloral" cuando tres o más especies se presentaron con porcentajes iguales o mayores al 10\% (Louveaxet al. 1978). Las mieles multiflorales se dividieron en "biflorales" cuando dos tipos de polen tuvieron porcentajes secundarios, "oligoflorales" cuando dominan dos o más taxa de una sola familia botánica con porcentajes secundarios de polen y "poliflorales" cuando tres o más tipos de polen se registraron con porcentajes secundarios (Ramírez-Arriaga et al. 2011).

Determinación del papel ecológico de A. mellifera L. Para conocer el papel ecológico de $A$. mellifera L. se utilizaron tres parámetros ecológicos:

a) Para determinar las estrategias de pecoreo de las abejas se utilizó el índice de diversidad de Shannon-Weaver (H') (1949), basándose en la diversidad del espectro polínico encontrado:

$$
\mathbf{H}^{\prime}=-\sum_{1}^{n} p i \ln p i
$$

Donde:

$\mathbf{H}^{\prime}$ es el índice de diversidad

$\boldsymbol{p i}$ es la proporción de cada tipo polínico $(\boldsymbol{i})$

In es el logaritmo natural.

b) Para determinar la uniformidad de pecoreo se empleó el índice de equidad (Pielou 1977) o uniformidad (J'). Los valores de J' se aproximan a 0 cuando los recursos fueron utilizados de manera heterogénea y si los recursos fueron explotados homogéneamente, entonces los valores se aproximan a 1. El índice de equidad se calculó empleando la fórmula:

$$
\mathbf{J}^{\prime}=\frac{\mathbf{H}^{\prime}}{\mathbf{H}^{\prime} \mathbf{m a x}}
$$

Donde:

$\mathbf{J}^{\prime}$ es la uniformidad de pecoreo de las abejas,

H'es el índice de diversidad

H'max es el logaritmo natural del total de número de tipos polínicos en la muestra (Kleinert \& Imperatriz 1987). 
c) Para la correlación se empleó el índice de Schoener (PS) que está basado en el porcentaje de similitud (Schoener 1968):

$$
\mathbf{P S}=-\frac{1}{2} \sum_{h}^{n}[p h i-p h j]
$$

donde:

PS es el recurso correlacionado,

phi es la proporción de granos de polen de la especie vegetal $\mathbf{h}$, en la muestra de la colonia i para el mes en cuestión

$\boldsymbol{h}$ especies de plantas visitadas por A. mellifera L. en la muestra $\boldsymbol{i}$ para ese periodo

$\boldsymbol{p h} \boldsymbol{j}$ es la proporción de granos de polen de la especie vegetal $\mathbf{h}$, en la muestra de la colonia $\mathbf{j}$ para el mes en cuestión.

\section{RESULTADOS}

Caracterización palinológica. Los análisis de 40 muestras de miel de Apis mellifera L. del estado de Tabasco, mostraron un total de 129 tipos polínicos, de los cuales 26 se identificaron a nivel de especie, 26 a nivel de género y 26 a nivel de familia (Cuadro 1, Figs. 2 a 7), el resto quedaron asignados a tipos morfológicos.

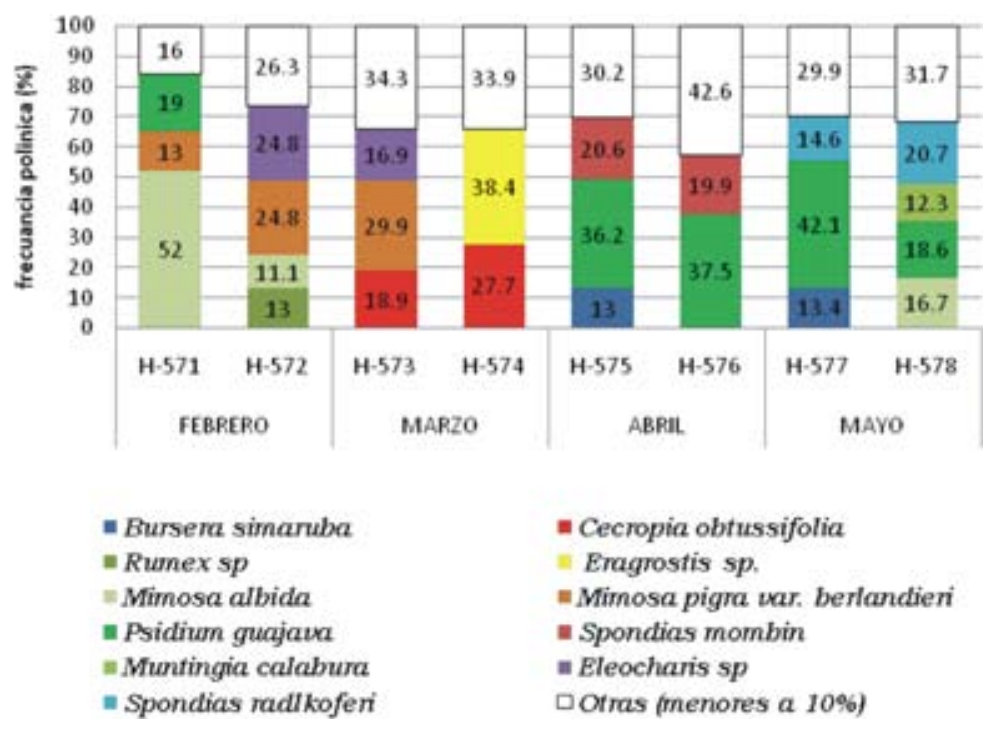

Figura 2. Especies de importancia ( $\geq 10 \%$ ) en mieles del municipio Centro, Tabasco, colectadas en 2009. 


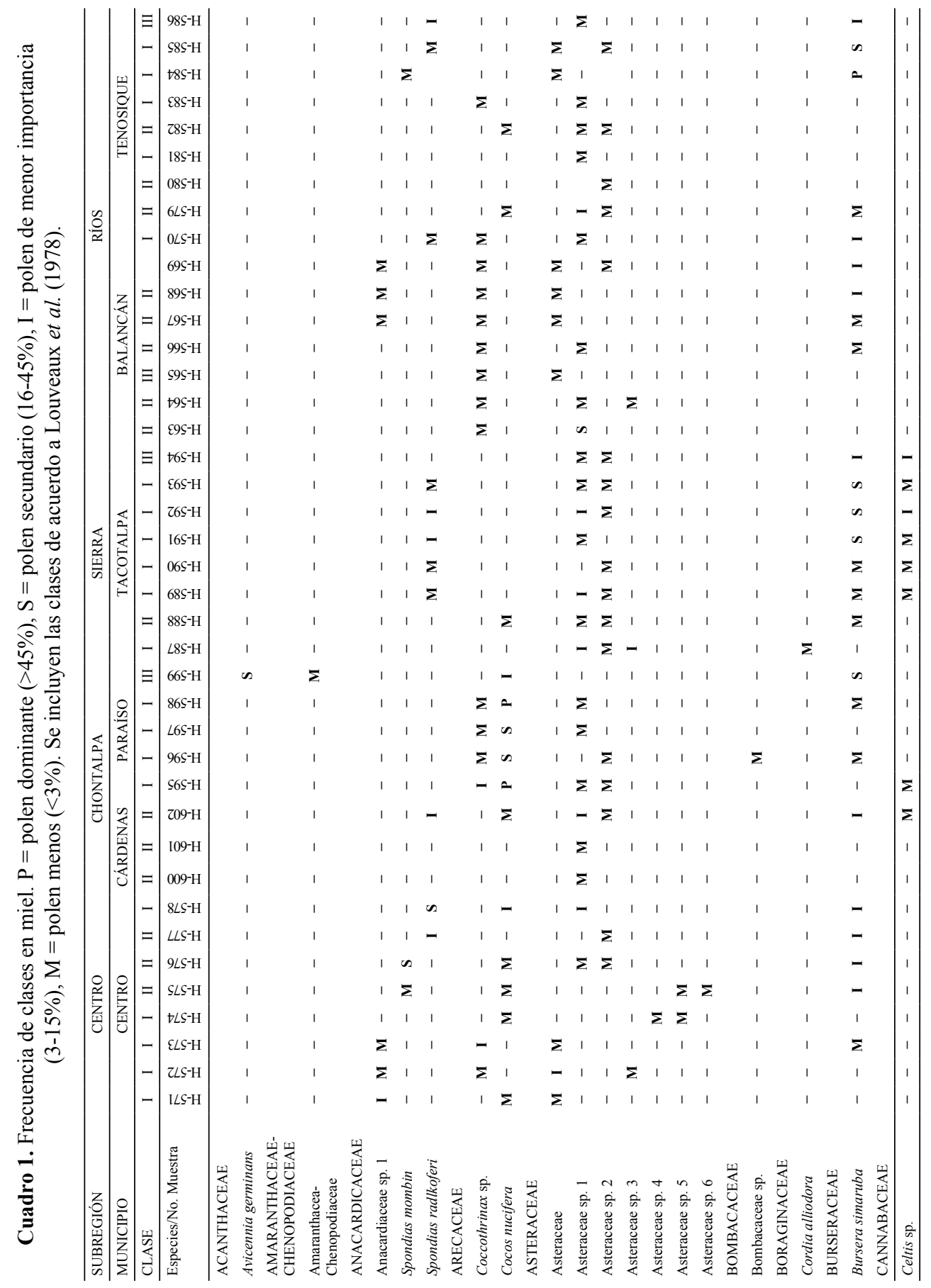




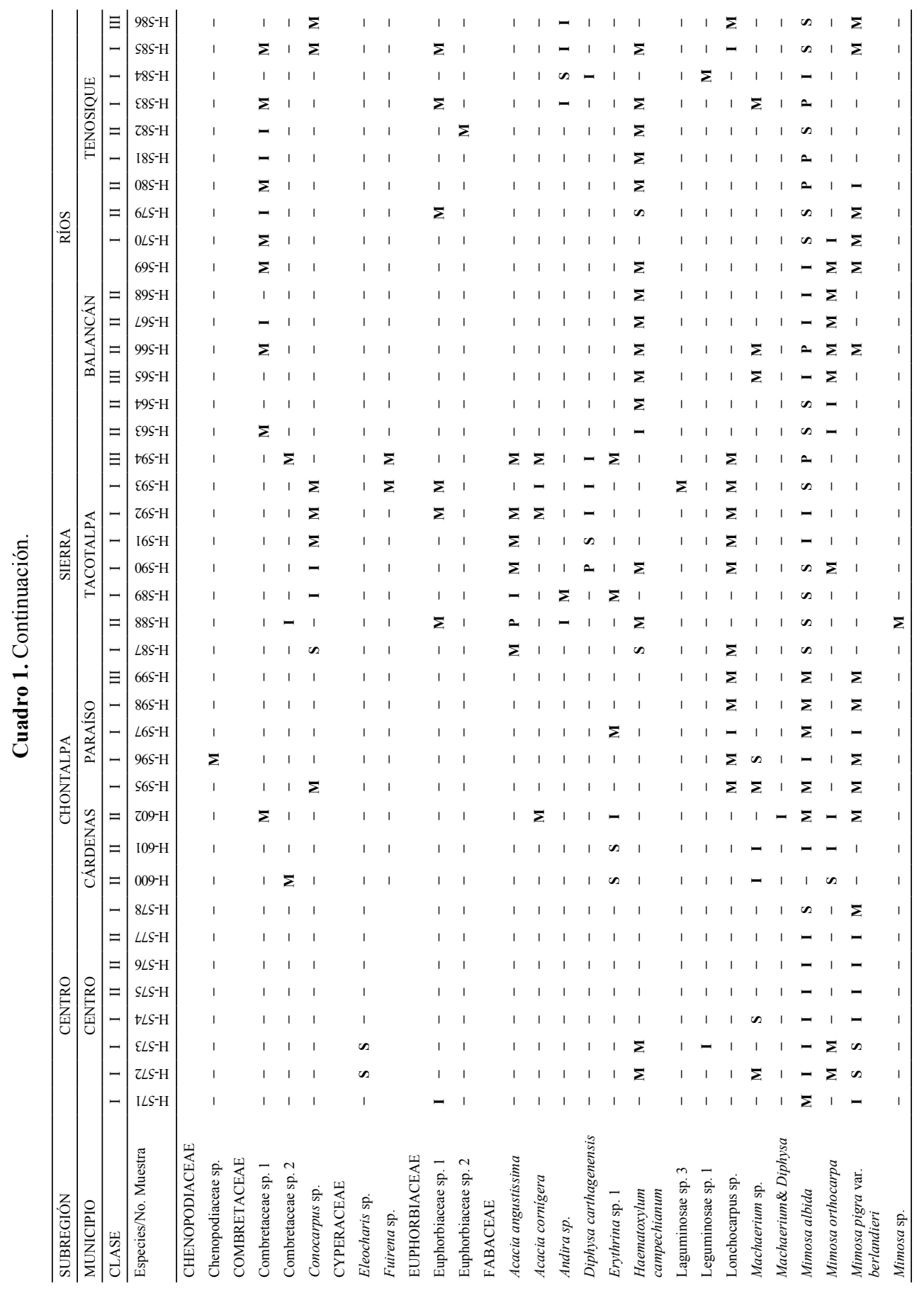




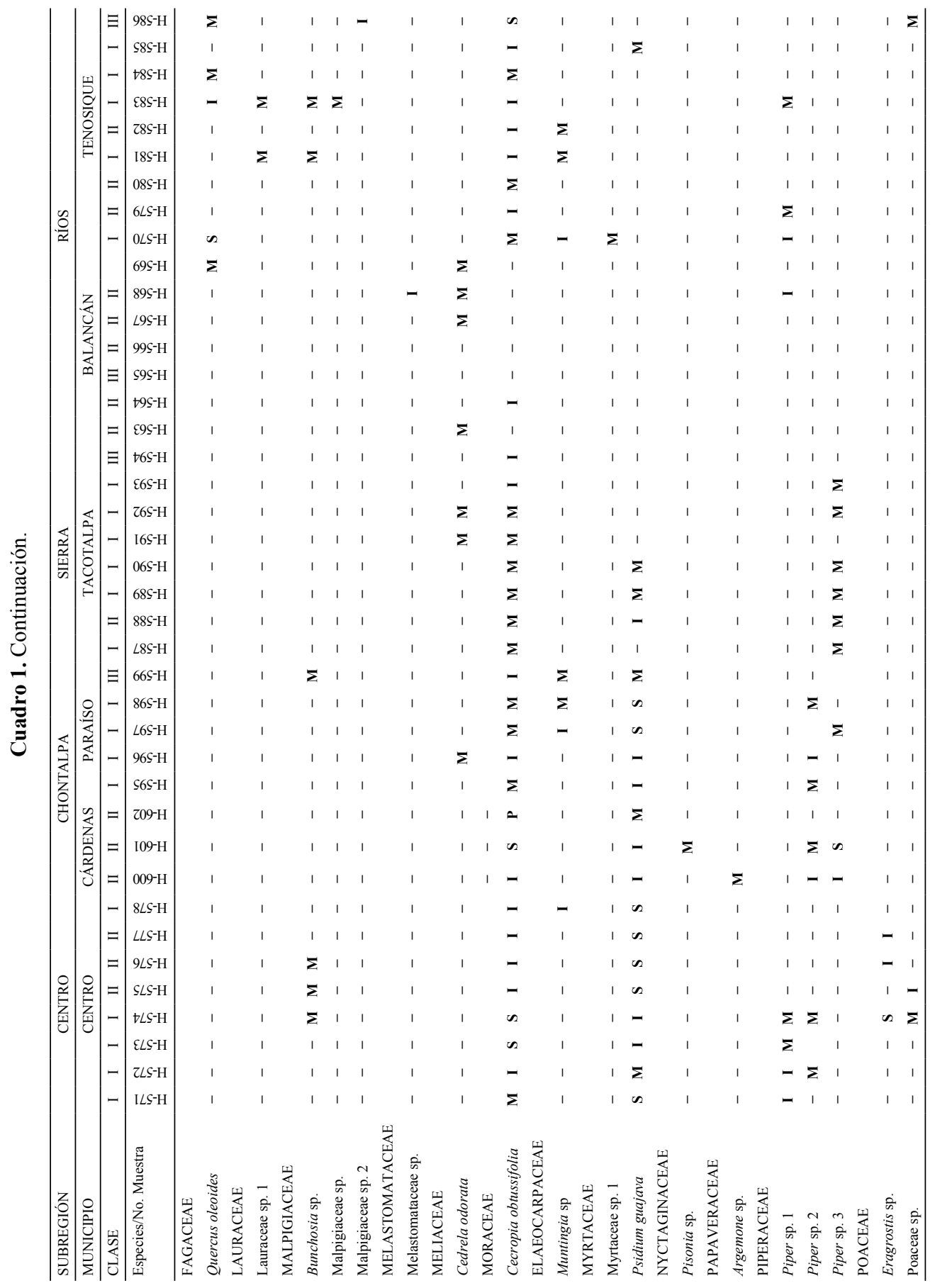




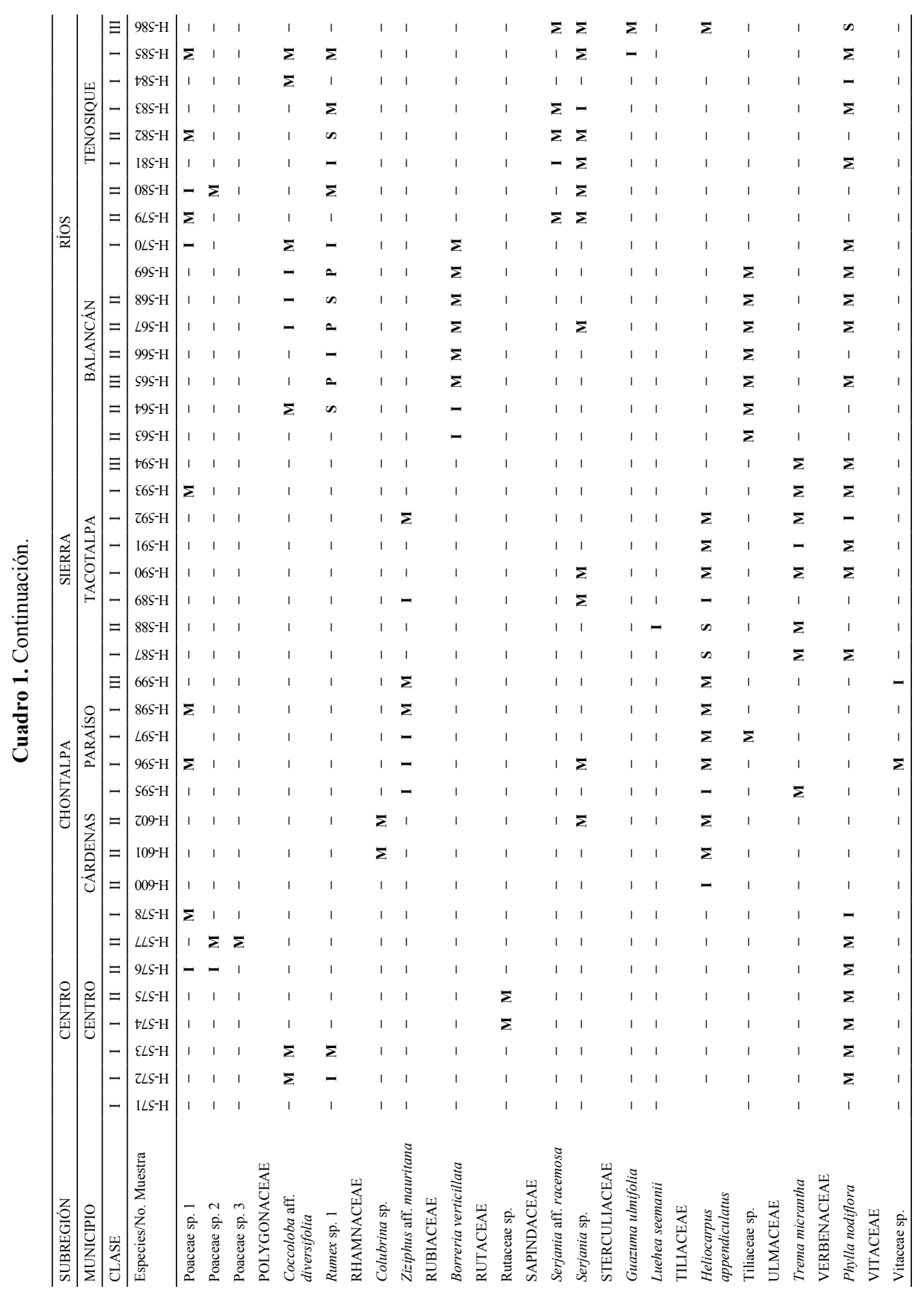


Es relevante resaltar que en las regiones estudiadas, las mieles de $A$. mellifera se caracterizaron con base a su espectro polínico: 14 muestras fueron monoflorales con dominancia de un tipo polínico con porcentajes $\geq 45 \%$, obteniéndose para la Subregión Centro, una miel monofloral de Mimosa albida en el municipio Centro (H571, Fig. 2); tres mieles monoflorales en la Subregión Chontalpa: una de Cecropia obtusifolia en el municipio de Cárdenas (H-602, Fig. 3) y dos de Cocos nucifera y M. albida respectivamente en el municipio de Paraíso (H-595 y H-598, Fig. 4); dos mieles monoflorales en la Subregión Sierra: de Diphysa carthagenensis y M. albida en el municipio de Tacotalpa (H-590 y H-594, Fig. 5) y ocho mieles monoflorales para la Subregión de los Ríos: cuatro para el municipio de Balancán de Rumex sp.1 (H-564, H-567 y H-599, Fig. 6) y M. albida (H-566, Fig. 6) y cuatro en el municipio de Tenosique de M. albida (H-580, H-581 y H-583, Fig. 7) y Bursera simaruba (H584, Fig. 7).

Además, siete mieles fueron clasificadas como biflorales: dos mieles para la Subregión Centro en el municipio del mismo nombre con las asociaciones Eragrostis sp. + C. obtusifolia, y Psidium guajava + Spondias mombin (H-574 y H-576, Fig. 2); una miel bifloral para la Subregión de la Chontalpa en el municipio de Paraíso de $C$. nucifera + P. guajava (H-597, Fig. 4); una miel para la Subregión de la Sierra en el municipio de Tacotalpa de M. albida + B. simaruba (H-593, Fig. 5), y tres mieles para la subregión de los Ríos, dos en el municipio de Balancán de M. albida + Rumex sp.1 y Quercus oleoides + Mimosa albida (H-564 y H-570, Fig. 6) y una del municipio de Tenosique con M. albida + Rumex sp.1 (H-582, Fig. 7).

Las mieles más abundantes fueron la multiflorales con un total de 19: cinco mieles en el municipio Centro, de la Subregión Centro (H-572, H-573, H-575, H-577 y H-578, Fig. 2); cuatro para la Subregión Chontalpa, dos en el municipio de Cárdenas (H-600 y H-601, Fig. 3) y dos en el municipio de Paraíso (H-596 y H-599, Fig. 4); cinco para la Subregión Sierra en el municipio de Tacotalpa (H-587, H-588, H-589, H-591 y H-592, Fig. 5) y cinco para la Subregión de los Ríos, dos en el municipio de Balancán (H-563 y H-568, Fig. 6) y tres en el municipio de Tenosique (H-579, H-585 y H-586, Fig. 7).

Considerando la diversidad total de palinomorfos, solamente 29 taxa fueron de importancia por presentarse con porcentajes $\geq 10 \%$ y representan el $20 \%$ del total (Ramalho et al. 1985): Avicennia germinans L. (Acanthaceae), Borreria verticillata L. (Rubiaceae), B. simaruba (L.) Sarg. 1890 (Burseraceae), C. obtusifolia Bertol. 1840 (Moraceae), Coccoloba aff. diversifolia Jacq. (Polygonaceae), Conocarpus sp. (Combretaceae), Rumex sp. 1 (Polygonaceae), Eleocharis sp. 1 (Cyperaceae), Eragrostis sp. (Poaceae), Asteraceae sp. 1 y sp. 2 (Asteraceae), Andira sp. (Fabaceae), D. carthagenensis Jacq. (Fabaceae), Erythrina sp. 1 (Fabaceae), H. campechianum L.(Fabaceae), H. appendiculatus Turcz.(Tiliaceae), Machaerium sp. (Fabaceae); M. albida Humb.y Bonpl. ex Willd (Fabaceae), M. pigra var. berlandieri (A. Gray. ex 


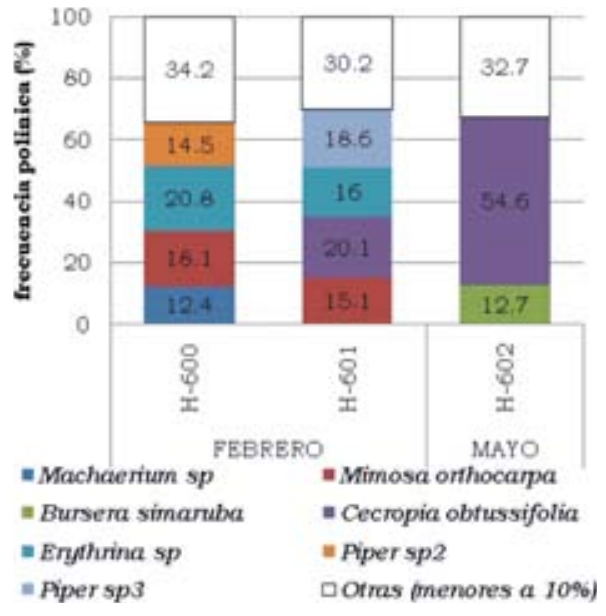

Figura 3. Especies de importancia ( $\geq 10 \%)$ en mieles del municipio Cárdenas, Tabasco, colectadas en 2009.

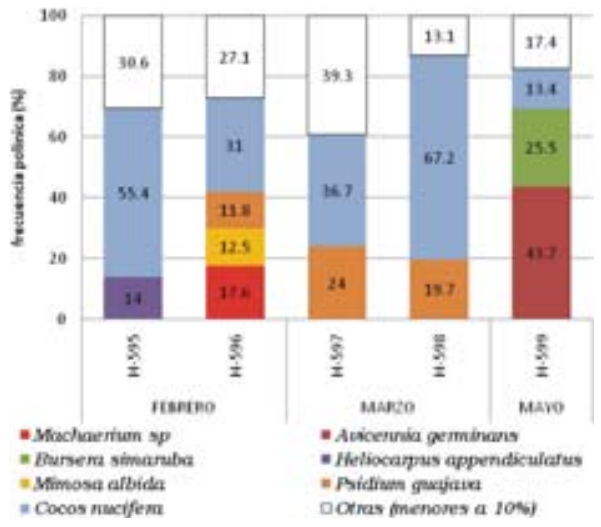

Figura 4. Especies de importancia ( $\geq 10 \%)$ en mieles del municipio Paraíso, Tabasco, colectadas en 2009.

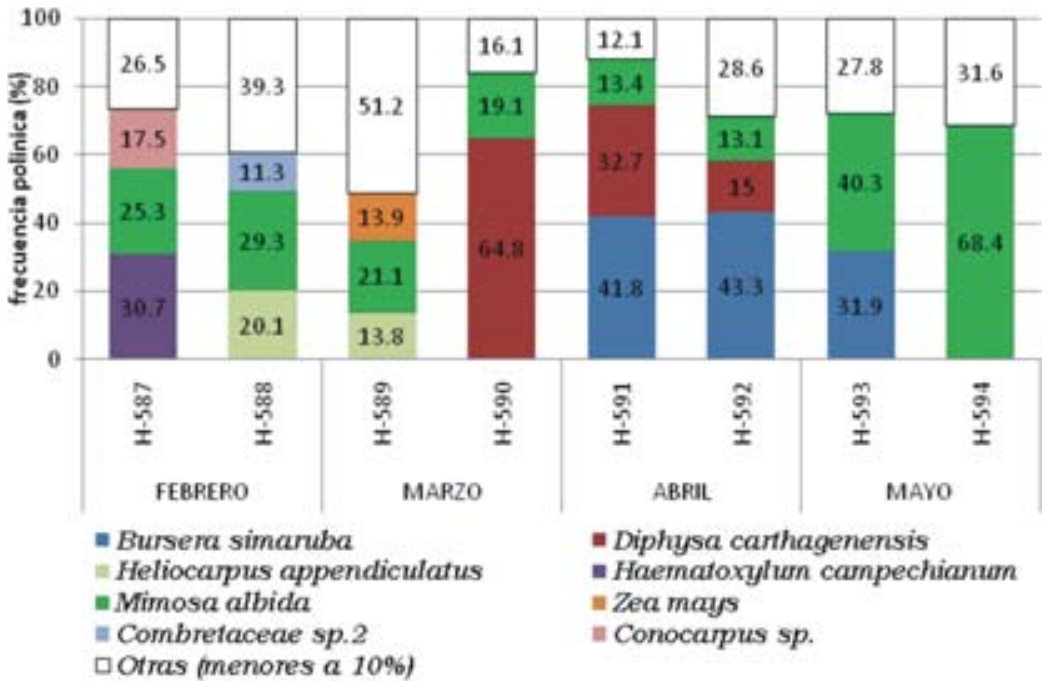

Figura 5. Especies de importancia ( $\geq 10 \%)$ en mieles del municipio Tacotalpa,

Tabasco colectadas en 2009. 
Castellanos-Potenciano et al.: Contenido polínico en mieles de abeja en Tabasco

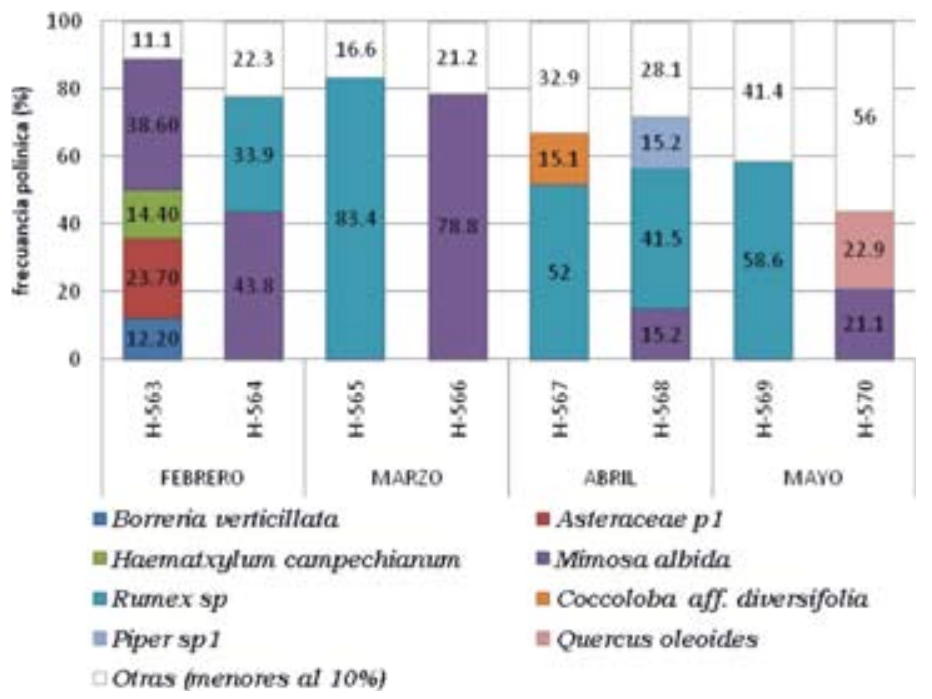

Figura 6. Especies de importancia ( $\geq 10 \%$ ) en mieles del municipio Balancán, Tabasco, colectadas en 2009.

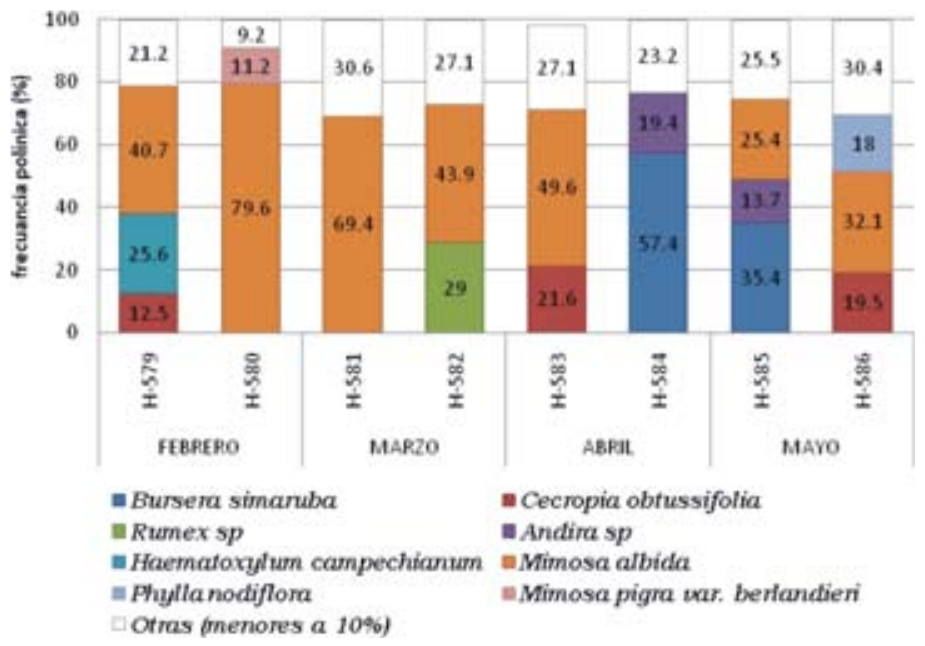

Figura 7. Especies de importancia ( $\geq 10 \%$ ) en mieles del municipio Tenosique, Tabasco, colectadas en 2009. 
Torr) B.L. Turner (Fabaceae), Phyla nodiflora (L.) Greene (Verbenaceae), Piper sp. 1, sp. 2 y sp. 3 (Piperaceae), Q. oleoides Cham. \&Schlecht (Fagaceae), S. mombin L. (Anacardiaceae), S. radlkoferi Donn. Sm. (Anacardiaceae), C. nucifera L. (Arecaceae), Muntingia sp. (Muntigiaceae) y la Poaceae Zea mays L. (Figs. 8 y 9).

La mayoría de los recursos que fueron explotados intensamente corresponden al estrato arbóreo (55.2\%): Machaerium sp., A. germinans, B. simaruba, C. obtusifolia,

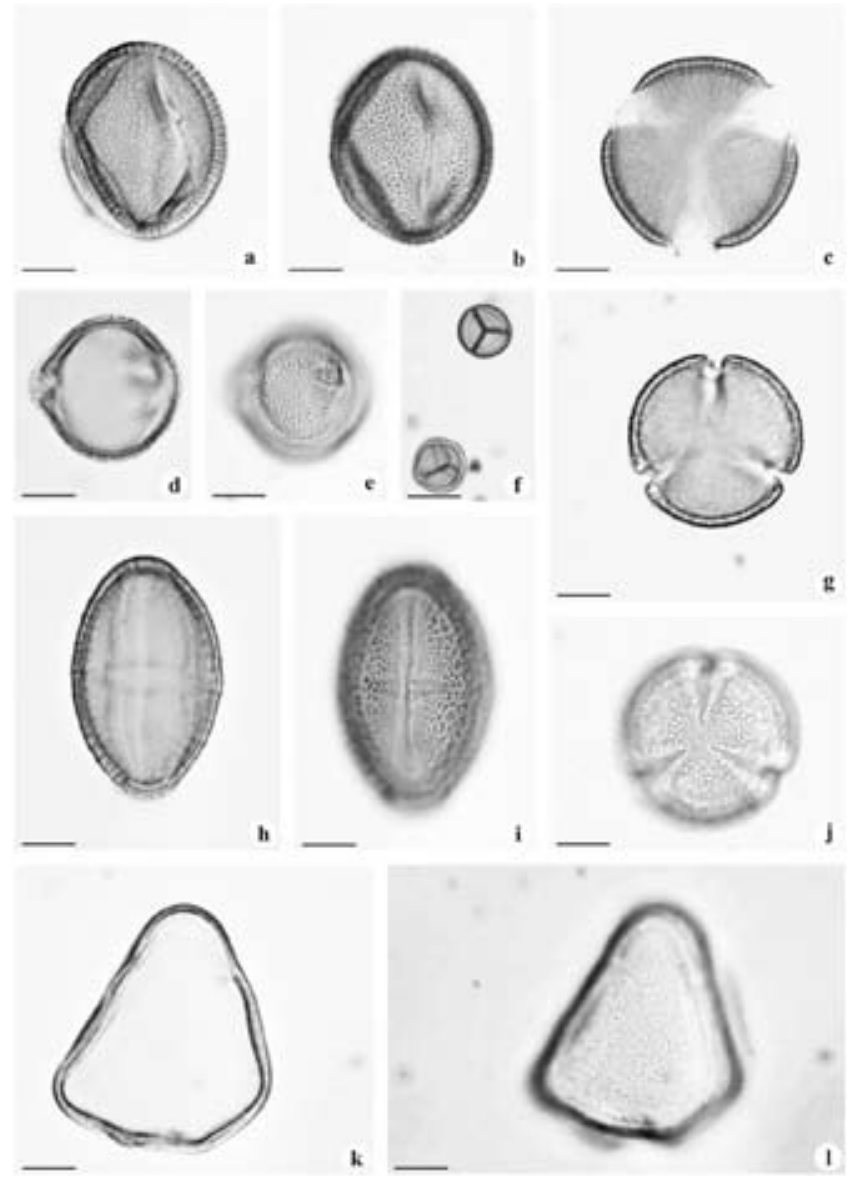

Figura 8. Polen de mieles de Tabasco con porcentajes $\geq 10 \%$ : Avicennia germinans (a-c); Bursera simaruba (d, e); Mimosa albida (f); Rumex sp. 1(g, j); Eleocharis sp.1 (k, 1); Heliocarpus appendiculatus $(\mathrm{h}, \mathrm{i})$. Todos los granos de polen fotografiados con objetivo 100X, la escala representa 10 micras. 


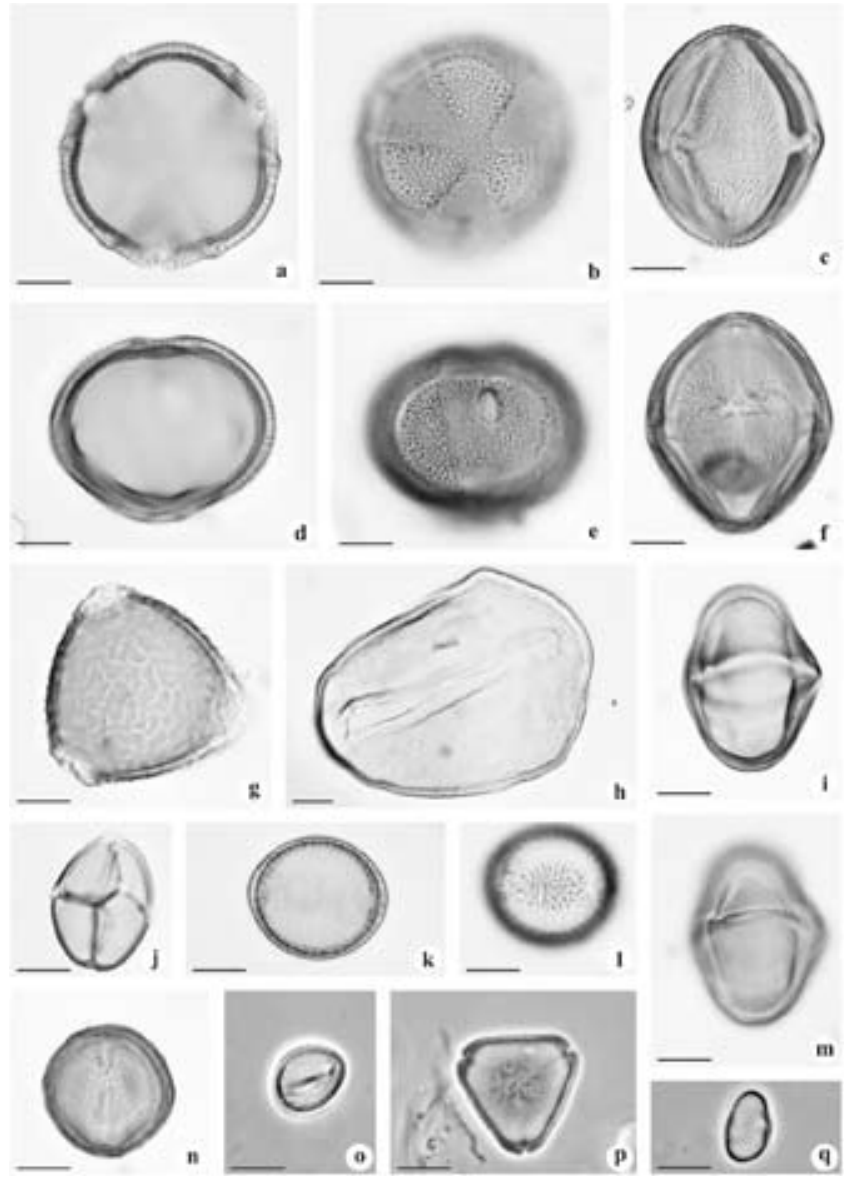

Figura 9. Polen de mieles de Tabasco con porcentajes $\geq 10 \%$ : Haematoxylum campechianum (a, b, d, e); Spondias radlkoferi (c);Spondias mombin (f); Erythrina sp. 1 (g); Cocos nucifera (h); Phyla nodiflora (i, m); Mimosa pigra var. berlandieri (j); Borreria verticillata (k, 1); Quercus oleoides (n); Piper sp. 2 (o); Psidium guajava (p); Cecropia obtusifolia(q). Todos los granos de polen fotografiados con objetivo $100 \mathrm{X}$, la escala representa 10 micras.

C. aff. diversifolia, D. carthagenensis, Erythrinasp.1, H. campechianum, H. appendiculatus, $P$. guajava, $Q$ oleoides, S. mombin, C. nucifera, Muntingia sp., Conocarpus sp. y $S$. radlkoferi.

El estrato arbustivo correspondió al $17.2 \%$ con la presencia de Andira sp., $M$. albida, M. pigra var. berlandieri, Piper sp. 1, sp. 2 y sp. 3 y finalmente, el estrato her- 


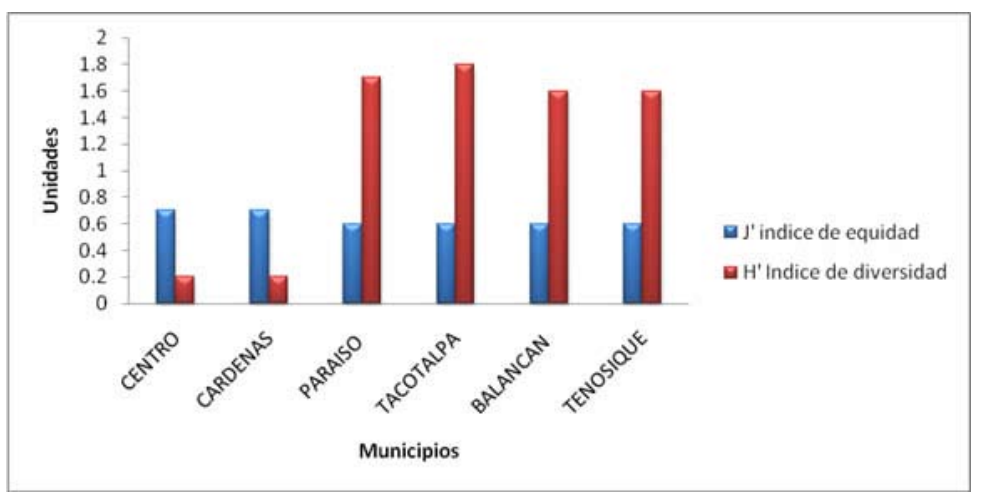

Figura 10. Valores promedio del índice de diversidad $\left(\mathrm{H}^{\prime}\right)$ e índice de equidad $\left(\mathrm{J}^{\prime}\right)$ registrados durante el período de muestreo en las cuatro subregiones apícolas.

báceo está representado en un $27.6 \%$ con Rumex sp. 1, Eragrostis sp., Asteraceae sp. 1 y sp. 2, P. nodilfora y Z. mays. El desplazamiento de las abejas hacia los diferentes estratos fue indistinto a los periodos de muestreo.

Análisis cuantitativo del polen. Considerando la cantidad absoluta de granos de polen, el mayor número de mieles $(\mathrm{n}=19)$ fue del grupo II, conteniendo de 20,000 a 100,000 granos de polen en diez gramos de miel, le sigue en importancia el grupo I con menos de 20,000 granos de polen $(n=18)$ y finalmente tres muestras fueron clasificadas en el grupo III con 100,000 a 500,000 granos de polen en 10 gramos de miel (Cuadro 1).

Determinación del papel ecológico de $\boldsymbol{A}$. mellifera $\mathbf{L}$. Por otro lado, la Figura 10 muestra los valores del índice de diversidad de Shannon-Weaver (H') y uniformidad (J') de las muestras de miel analizadas. De acuerdo con el número de taxa que visitó A. mellifera en los seis municipios y la frecuencia de aparición de los tipos polínicos, los mayores valores del índice de diversidad se registraron en las muestras de Balancán (2.6), Tacotalpa (2.5), Centro (2.2) y Cárdenas (2.2) donde se encontró que el comportamiento de esta especie de abeja es poliléctico al colectar néctar de varias especies vegetales.

Los mayores valores de $\mathrm{J}^{\prime}$ se registraron en Centro (0.8) y Tacotalpa (0.8) indicando un pecoreo más homogéneo, mientras que en las muestras con bajo índice de equidad mostraron una explotación heterogénea debido a las preferencias florales sobre pocos recursos (Fig. 10). El índice de correlación varió de 0.024 a 0.980 . Los mayores valores de PS se registraron entre Tenosique y Tacotalpa (0.89) cuando Apis colectó néctar de $B$. simaruba, D. carthagenensis y M. albida. 


\section{DISCUSIÓN}

El análisis de los parámetros ecológicos basados en las frecuencias de los granos de polen contenidos en la miel de $A$. mellifera mostró un comportamiento poliléctico, ya que durante los cuatro meses de muestreo visitó un gran número de especies vegetales $(\mathrm{n}=128)$, pero solo 29 fueron de real importancia sensu Ramalho et al. (1985).

En la familia Anacardiaceae, se registraron dos especies: S. mombin y S. radlkoferi, de ellas, solo $S$. mombin, conocido comúnmente como "jobo roñoso", ha sido reportado como parte de la flora melífera de México (Espina \& Ordetx 1983), del estado de Tabasco por la SAGARPA (2004) y Oaxaca (Santos-Ramos 2008). Esta especie presenta amplia distribución en todo el estado de Tabasco, ha sido citada por Cárdenas (1985) para la subregión de la Chontalpa como especie presente en miel y por Córdova et al. (2009) como elemento importante en miel madura.

De la familia Arecaceae, C. nucifera es considerada como una de las especies con mayor valor apícola, ya que aporta néctar y polen en cantidades importantes para el pecoreo de las obreras para la subregión Chontalpa (Cárdenas 1985; SAGARPA 2004; Santos-Ramos 2008; Pascual 2008) y para la subregión Pantanos del estado de Tabasco (Córdova 2009). Las mieles de coco son muy características por su sabor fuerte, ligeramente ácido, de color obscuro, en ciertas zonas el color de la miel extraída es de color amarillo verdoso (Espina \& Ordetx 1983). Según Espina \& Ordetx (1983), Cárdenas (1985), SAGARPA (2004) y Santos-Ramos (2008) la floración de esta especie es irregular, puede presentarse todo el año.

Asteraceae es una familia cuyos tipos polínicos son muy similares entre las especies, lo que dificulta su diferenciación. A no ser que se cuente con una colección palinológica de referencia muy completa de todas las especies de compuestas que se encuentren en el área del apiario, su determinación a nivel específico es difícil, por lo que se asignan tipos morfológicos diferenciados por el tamaño de sus espinas, distancia entre ellas y la forma del grano de polen. Como lo reporta Espina \& Ordetx (1983) es una familia muy diversa, con numerosas especies nectaríferas y poliníferas, además, es una de las familias más grandes, que comprende la décima parte de todas las plantas conocidas y en este trabajo estuvo representada tanto en número de especies (7) como en frecuencia de aparición (Quiroz \& Arreguín 2008).

La especie $B$. simaruba (palo mulato) es un recurso melífero importante, su distribución es muy amplia, casi en toda la República Mexicana. En este trabajo se encontró una miel monofloral de $B$. simaruba en el municipio de Tenosique, de la Subregión de los Ríos. El palo mulato ha sido reportado por la SAGARPA $(1998,2002$, 2003 y 2004) en los listados de flora melífera, por Cárdenas (1985) para la subregión Chontalpa en Tabasco; Villanueva (2002) dio a conocer la especie para la Península de Yucatán; Martin (2006) para todo México; Navarro (2008) y Santos-Ramos (2008) en Oaxaca y Córdova (2009) en Tabasco. Según Martin (2006) un inconveniente para esta especie, es que debido a su amplia distribución en el país, dificulta el 
poder denominar a una miel por su origen geográfico y aunque fuera más abundante en un lugar que en otro, presentaría complicaciones al pretender una denominación de origen solo por la presencia de dicha especie. Esta especie crece en una variedad de hábitats de selvas bajas sub-deciduas y selvas altas siempre verdes, produce néctar y polen y es utilizado para la construcción de cercos vivos (Carvajal 2005), casas, en carpintería y como remedio en la medicina (Ramírez-Arriaga et al. 2011).

La familia Elaeocarpaceae estuvo representada por Muntingia calabura, especie reportada por Espina \& Ordetx (1983) para Latinoamérica y no se encontraron citas de la especie como parte de la flora melífera del estado.

En la familia de las Fabaceae se determinaron M. albida, M. pigra var. berlandieri, H. campechianum, D. carthagenensis, Erythrina sp.1, Machaerium sp.y Andira sp. como taxa de importancia. La especie $M$. albida está citada en la flora apícola de los estados de Chiapas (SAGARPA 2002) y Veracruz (SAGARPA 2003). De estas leguminosas, $M$. albida ha sido descrita como una especie abastecedora de polen, sin embargo aún cuando no presenta nectarios, es probable que produzca néctar en una zona poco diferenciada (Lewis \& Elias 1981). Esta planta, por su frecuencia en las muestras se considera de gran importancia alimenticia, coincidiendo con los reportes de Espina \& Ordetx (1983). En Sudamérica, Girón (1995) reafirma la importancia de la especie en Colombia, así como Carmello et al. (2000) para la bahía de Brasil.

En el caso de M. pigra var. berlandieri se encontró descrita por Córdova et al. (2009) en mieles de Tabasco; Navarro (2008) la observó en mieles de Oaxaca y también se ha citado en la flora apícola de Veracruz (SAGARPA 2003) y Espina \& Ordetx (1983) la reportan como especie polinífera.

En cuanto a H. campechianum (Palo de tinto), es una especie descrita con crecimiento rápido y de fácil propagación. Espina \& Ordetx (1983) mencionan que es una de las especies que produce más miel en el mundo, siempre y cuando las condiciones de humedad sean las idóneas. Además, se ha citado para el estado de Veracruz (SAGARPA 2003) otra especie del mismo género, $H$. brasiletto.

D. carthagenensis, es una especie nectarífera de importancia apícola en la Península de Yucatán y en el trópico en general (Espina \& Ordetx 1983; SAGARPA 1998). Por otro lado, el taxón Erythrina es de escaso valor apícola, por la producción inconstante de néctar y por las flores alargadas en forma de cuchillo, que dificultan a las abejas el acceso hacia los nectarios (Espina \& Ordetx 1983).

Q. oleoides (Fagaceae) es muy importante para América del Norte (Espina \& Ordetx1983), sus especies son consideradas poliníferas, ya que la producción de néctar es mínima, por lo que las abejas pecorean este recurso para alimento larval. Según Ortiz (1990) y Mielso (2008) en zonas donde hay un aporte mayor de agua, los árboles de Quercus producen una gran cantidad de savia dulce que sale por todos los poros disponibles, el cual se puede volver importante para las abejas, quienes recogen esta "mielada" y la procesan igual que el néctar floral. Aunque no hay informes 
específicos de $Q$. oleoides en el estado, puede tratarse de una especie tanto de valor polinífero como productor de mielada.

La familia Moraceae estuvo representada por la especie C. obtusifolia (Guarumo) la cual no se encontró en los listados de flora melífera del estado. Martínez-Hernández et al. (1993) reporta la especie en miel de Trigona en la región de Tacaná, Chiapas. La planta posee nectarios extraflorales que aprovechan las abejas, su presencia en mieles como elemento importante ya ha sido citada por Cárdenas (1985) y Córdova (2009).

En México la familia Poaceae ha sido descrita como parte de la miel en el estado de Chiapas (Ramírez-Arriaga 1989), Tabasco (Cárdenas 1985) y del valle de México (Piedras \& Quiroz 2007). También se tienen registros de observaciones de campo en los estados de Chiapas, Veracruz y Tabasco (SAGARPA 2002, 2003 y 2004), destacándose Zea mays como especie polinífera y nectarífera (azúcares que se acumulan en la base de las hojas), coincidiendo con lo publicado años antes por Espina \& Ordetx (1983). Además, Sajwani et al. (2007) cita que a pesar de tratarse de una especie con polinización anemófila, es posible encontrarla en la miel por su aporte polinífero.

Psidium guajava (Myrtaceae) se considera como parte de la flora nectaropolinífera para la zona de Chontalpa por Cárdenas (1985) y elemento importante en la miel por Córdova et al. (2009). Es una especie ampliamente distribuida en las zonas cálidas de México formando malezas ó como extensiones de monocultivo, debido al interés comercial que tiene el fruto. Espina \& Ordetx (1983) la describen como uno de los mayores productores de polen en la primavera y dudan de sus aportes nectaríferos, por la falta de observación durante el pecoreo.

La Familia de las Piperaceae estuvo representada por tres tipos del género Piper, debido a la similitud de los granos de polen del género y a que las diferencias entre los granos de polen de una especie a otra solo son observables en el Microscopio Electrónico de Transmisión, no fue posible determinar los granos a nivel de especie, por lo que solo se diferenciaron en base al tamaño del grano y a la ornamentación de la exina (Ibarra \& Martínez-Hernández 1997). Este género fue reportado por MartínezHernández et al. (1993) en muestras de polen de Scaptotrigona mexicana y en miel y polen de Trigona (Tetragonisca) angustula. Debido a la inflorescencia que posee esta planta, no permite la formación de nectarios, por lo que se considera como una especie sustancialmente de valor polinífero (Adams \& Smith 1981; Sajwani et al.2007).

En las Polygonaceae encontramos $C$. aff. diversifolia y Rumex sp. 1, que ya han sido citadas previamente como flora apícola (Espina \& Ordetx 1983). El género Coccoloba es considerado de importante valor por su gran aporte nectarífero en las cosechas de miel (Espina \& Ordetx 1983).

La familia Rubiaceae estuvo representada por la especie $B$. verticillata, el género se ha encontrado en miel de abejas nativas Plebeia sp. (Ramírez-Arriaga 1989), también se ha registrado en mieles de A. mellifera en Sudamérica (Sodré et 
al. 2007). Espina \& Ordetx (1983) lo reportan como de interés apícola para toda América.

Dentro de la familia Tiliaceae, $H$. appendiculatus (jolotzin) es considerada en la apiflora del estado de Chiapas (SAGARPA 2002). Otra especie, del mismo género $H$. donnell-smithii, es comúnmente aprovechada por A. mellifera (Martínez-Hernández et al. 1993; Ramírez-Arriaga \& Martínez-Hernández 2007; Quiroz \& Arreguin 2008; Navarro 2008; Díaz 2008).

Es importante destacar que se cuenta con nuevos reportes de recursos nectaríferos para A. mellifera: S. radlkoferi (Anacardiaceae), Conocarpus sp. (Combretaceae), $P$. nodiflora (Verbenaceae) y Machaerium sp. (Fabaceae).

De una forma amplia, se puede observar, que algunas especies aunque son catalogadas como poliníferas, como las Poaceae, Moraceae y Piperaceae, su presencia en la miel según Adams \& Smith (1981) es atribuible a que cuando las abejas colectan el néctar, llevan consigo las cargas de polen curbicular, por lo cual el polen de dichas especies se mezcla con el néctar libado. Sin embargo, es importante considerar la presencia de nectarios extraflorales como el caso de C. obtussifolia y Z. mays, o la presencia de una estemozona donde se produce néctar en Mimosa (Lewis \& Elias 1981), además de la producción de mieladas de Quercus, por lo que estos elementos si proveen recursos nectaríferos y no deben ser considerados exclusivamente como poliníferos.

En general, A. mellifera registró valores de diversidad $\left(\mathrm{H}^{\prime}\right)$ que confirman sus hábitos generalistas. De forma similar y de acuerdo a la literatura, dependiendo de la vegetación disponible será el pecoreo de la abeja (Girón 1995). Los valores de equidad mostraron que cuando $A$. mellifera explota un menor número de especies de plantas, el uso de los recursos es más heterogéneo, comportamiento que se observó en cuatro localidades (Figura 10). De forma inversa, cuando la abeja común pecoreó sobre una mayor diversidad de especies de plantas, el uso de los recursos fue más homogéneo, similar al comportamiento de pecoreo ya descrito para A. mellifera (Girón 1995; Piedras \& Quiroz 2007; Díaz 2008; Navarro 2008).

Se registraron altos índices de correlación entre localidades donde los mismos elementos nectaríferos fueron explotados de forma similar, como lo fue cuando $A$. mellifera se desplazó sobre las especies B. simaruba, C. obtusifolia y M. albida.

Agradecimientos. Blanca Patricia Castellanos Potenciano es egresada de la Maestría en Ciencias en Producción Agroalimentaria en el Trópico y ex becaria del Consejo Nacional de Ciencia y Tecnología (CONACYT). Los autores agradecen a los apicultores cooperantes: Rufo Peralta Jacinto, Fernando Martínez Gómez; Carlos Cárdenas Jiménez y Límbert Zurita Cruz, así como a los trabajadores de los apiarios del Colegio de Postgraduados, Campus Tabasco. Especial agradecimiento al Dr. Enrique Martínez Hernández responsable del Laboratorio de Palinología del Instituto de Geología de la UNAM por haber brindado las facilidades para el uso de las instalaciones y por la asesoría brindada en esta investigación. 


\section{LITERATURA CITADA}

Acosta-Castellanos, S. \& R. Palacios Chávez. 2001. Plants of apicultural interest in the Pluma Hidalgo zone, Oaxaca, Mexico, pp. 459-469 In: Goodman, D. K. and R. T. Clarke. (Eds.). Proceedings of the IX International Palynological Congress. Houston, Texas, U.S.A., 1996; American Association of Stratigraphic Palynologists Foundation.

Adams, R. J. \& M. V. Smith. 1981. Seasonal pollen analysis of nectar from the hive and of extracted honey. Journal of Apicultura Research, 20: 243-248.

Ayala, A. M. E. 2001. La apicultura de Península de Yucatán: un acercamiento desde la ecología humana. Centro de Investigaciones y de Estudios Avanzados del I.P.N, Unidad Mérida. Tesis de Maestría 153 pp. (Inédita).

Cárdenas, Ch. 1985. Caracterización del ciclo apícola y flora nectarífera y polinífera, en la Chontalpa Tabasco, México. Colegio Superior de Agricultura Tropical. Tesis de Licenciatura 120 pp. (Inédita).

Carmello, M. A. C., C. A. Lopez de Carvalho, L. C. Marchini \& C. Fernandez de Olivera. 2000. Espectro Polinico de Amostras Demel de Apis mellifera L., Coletadas NaBahia. Botânica E Fisiologia Vegetal. Bragantia Campinas, 59: 1-6.

Carvajal, J. 2005. Establecimiento de postes de Chacah (Bursera simaruba, L. Sarg) como cerco vivo. Livestock Research for Rural Development, 17(2).

Córdova, C. 2009. Determinación Geográfica y Botánica de miel de abeja (Apis mellifera L.) del estado de Tabasco, México. Colegio de Postgraduados, Institución de Enseñanza e Investigación en Ciencias Agrícolas. Programa de Postgrado en Producción Agroalimentaria en el Trópico. Tesis de Maestría en Ciencias 147 pp. (Inédita).

Córdova-Córdova, C., E. Ramírez-Arriaga, E. Martínez-Hernández \& J. M. Zaldívar-Cruz. 2009. Caracterización botánica de la miel de abeja (Apis mellifera L.) de cuatro regiones del estado de Tabasco, México, mediante técnicas melisopalinológicas. (En prensa).

Díaz, E. 2008. Estudio Palinológico y Fisicoquímico de la miel de Apis mellifera L. del municipio de San Pedro Tapana, Oaxaca. Escuela de Medicina Veterinaria y Zootecnia. Universidad Autónoma Benito Juárez de Oaxaca. Tesis de Licenciatura. 81 pp. (Inédita).

Erdtman, G. 1969. Handbook of Palynology - An Introduction to the Study of Pollen Grains and Spores. Munksgaard, Copenhagen.

Espina, D. \& G. Ordetx. 1983. Flora apícola tropical. Editorial Tecnológica de Costa Rica. Costa Rica.

Girón, M. 1995. Análisis palinológico de la miel y la carga de polen colectada por Apis mellifera en el Suroeste de Antioquía, Colombia. Boletín del Museo de Entomología de la Universidad del Valle, 3: $35-54$.

Ibarra, G. \& E. Martínez-Hernández. 1997. Estudio palinológico de Ficus, subgénero Pharmacosycea (Moraceae) Veracruz, México. Boletín de la Sociedad Botánica de México, 61: 95-100.

Kleinert, A. \& V. L. Imperatriz. 1987. Aspects of the trophic niche of Melipona marginata marginata Lepeletier (Apidae, Meliponinae). Apidologie, 18: 69-100.

Lewis, G. P. \& T. S. Elias. 1981. Tribu Mimoseae, pp. 155-168. In: Polhill, R. M. and Raven, P. H. (Eds.). Advances in Legume Systematics. Part 1. Royal Botanic Gardens Kew, England.

Loayza, A. \& R. Ríos. 1999. Características del néctar y visitas de insectos a flores de Nicotiana glauca L. (Solanaceae): ¿Asociadas a cambios de temperatura y humedad del ambiente? Ecología en Bolivia, 33: 51-66.

Louveaux, J., Maurizio, A. \& G. Vorwohl. 1978. Methods of Melissopalinology. Bee World, 59: 39157. 
Maher, L. J. 1981. Statistics for microfossil concentration measurements employing samples spiked with marker grains. Review of Palaeobotany and Palynology, 32: 153-191.

Martin, P. 2006. La importancia de la melisopalinología en la apicultura y el comercio. Apitec, 58: 3-5.

Martínez-Hernández, J., E. Cuadrillero, O. Téllez, E. Ramírez-Arriaga, S. Sosa, J. Melchor, M. Medina \& M. Lozano. 1993. Atlas de las plantas y el polen utilizados por las cinco especies principales de abejas productoras de miel en la región del Tacaná, Chiapas, México. Instituto de Geología. Universidad Nacional Autónoma de México. México. 105 pp.

Martínez-Hernández, E. \& E. Ramírez-Arriaga. 1997. El polen que contiene la miel como indicador del origen floral y autenticidad de lotes comerciales. XI Seminario Americano de Apicultura. SAGAR y U.N.A. 7-10 de agosto de 1997 Acapulco, Guerrero, México.

Maurizio, A. 1939. Untersuchungen zur quantitativen Pollen-analyse des Honigs. Mitteilungen ausdem Gebiete der Lebensmitteluntersuchung und Hygiene, 30: 27-69.

Mielso. 2008. Mielada (Mielato) de Encina o Roble (Quercus sp.). http://www.mielso.es/archivos/pdf/ productos/floresta/miel_de_encina.pdf.

Müller, A. 1996. Host-plant specialization in western Palearctic Anthidiine bees (Hymenoptera: Apoidea: Megachilidae). Ecological Monographs, 66: 235-257.

Navarro, A. 2008. Estudio Palinológico y Fisicoquímico de la Miel de Apis mellifera L., en la Región Costa de Oaxaca: Distritos Jamiltepec, Juquila y Pochuta. Escuela de Medicina Veterinaria y Zootecnia. Universidad Autónoma Benito Juárez de Oaxaca. Tesis de Licenciatura. 117 pp. (Inédita).

Ortiz, P. L. 1990. Contribución al conocimiento de la flora apícola gaditiana. Lagascalia, 16: 199210.

Pascual, G. J. 2008. Caracterización fisicoquímica y polínica de las mieles del estado de Tabasco. Instituto Tecnológico Superior de Las Choapas. Ingeniería en Industrias Alimentarias. Tesis de Licenciatura. 117 pp. (Inédita).

Piedras, B. \& D. Quiroz. 2007. Estudio melisopalinológico de dos mieles de la porción sur del Valle de México. Polibotánica, 23: 57-75.

Pielou, E. C. 1977. Mathematical ecology. 2nd. Ed. Wiley-Interscience Publication, John WILEY \& Sons, Inc. New York, United States of America.

Porter, L. 2001. Landscape ecology of apiculture in the Maya area of La Montaña, Campeche, México. Universidad de Florida. Tesis de Doctorado 196 pp. (Inédita).

Price, P. 1997. Insect Ecology. John Wiley \& Sons, Inc. United States of America.

Quiroz-García, D. L. \& M. L. Arreguín-Sánchez. 2008. Determinación Palinológica de los recursos florales utilizados por Apis mellifera (Hymenoptera: Apidae) en el estado de Morelos, México. Polibotanica, 26: 159-173.

Ramalho, M., V. I. Imperatriz, A. Kleinert \& M. Cortopassi. 1985. Exploitation of floral resources by Plebeiaremota Holmberg (Apidae, Meliponinae). Apidologie, 16: 307-330.

Ramírez-Arriaga, E. 1989. Explotación de recursos florales por Plebeia sp. (Apidae) en dos zonas con diferente altitud y vegetación en el Soconusco Chiapas. Facultad de Ciencias. Universidad Nacional Autónoma de México. Tesis de Licenciatura 159 pp. (Inédita).

Ramírez-Arriaga, E. \& E. Martínez-Hernández. 2007. Melitopalynological Characterization of Scaptotrigona Mexicana Guérin (Apidae: Meliponini) and Apis mellifera L. (Apidae: Apini) Honey Samples in Northern Puebla State, México. Journal of the Kansas Entomological Society, 80: 377391.

Ramírez-Arriaga, E., Navarro-Calvo, L. \& E. Díaz-Carbajal. 2011. Botanical characterization of Mexican honeys from a subtropical region (Oaxaca) based on pollen analysis. Grana, 50: 40-54. 
Ruiz-Navajas, Y., M. Viuda-Martos, J. Fernández-López, J. M. Zaldívar-Cruz, V. Kuri \& J. A. Pérez-Álvarez. 2011. Antioxidant activity of artisanal honey from Tabasco, Mexico. International Journal of Food Properties, 14: 459-470.

Sajwani A., Farooq S. A., Patzelt A., Eltayeb E. A. \& V.M. Bryant. 2007. Melissopalynological studies from Oman. Palynology, 31: 63-79.

Santos-Ramos, De los M. 2008. Melisopalinología y determinación anual de los recursos nectaro-poliniferos en Apis mellifera scutellata Lep. en la Costa de Oaxaca en diferentes tipos de vegetación y agroecosistemas. Facultad de Ciencias. Universidad Nacional Autónoma de México.Tesis de Maestria. 57 pp. (Inédita).

Schoener, T.W. 1968. The Anolis lizards of Bimini: resource portitioning in a complex fauna. Ecology, 49: 704-726.

Secretaría de Agricultura, Ganadería, Desarrollo Rural, Pesca y Alimentación (SAGARPA). 1998. Flora Nectarifera y Polinifera en la Península de Yucatán. SAGARPA.

Secretaría de Agricultura, Ganadería, Desarrollo Rural, Pesca y Alimentación (SAGARPA). 1999. Flora Nectarifera y Polinifera del estado de Michoacán. SAGARPA.

Secretaría de Agricultura, Ganadería, Desarrollo Rural, Pesca y Alimentación (SAGARPA). 2002. Flora Nectarifera y Polinifera en el estado de Chiapas. SAGARPA.

Secretaría de Agricultura, Ganadería, Desarrollo Rural, Pesca y Alimentación (SAGARPA). 2003. Flora Nectarifera y Polinifera en el estado de Veracruz. SAGARPA.

Secretaría de Agricultura, Ganadería, Desarrollo Rural, Pesca y Alimentación (SAGARPA) 2004. Flora Nectarifera y Polinifera en el estado de Tabasco. SAGARPA.

Shannon, C. E. \& W. Weaver. 1949. The mathematical theory of communication. Univ. of Illinois Press, Urbano.

Simó, E. 2002. Las abejas de la miel y la polinización. Métode, 33: 72-76.

Sodré Da S., L. Marchini, C. Carvalho \& A. Moreti. 2007. Pollen analysis in honey samples from the two main producing regions in the Brazilian northeast. Annals of the Brazilian Academy of Sciences, 79: 381-388.

Stockmarr, J. 1971. Tablets with spores used in absolute pollen analysis. Pollen et Spores, 13: 615621.

Villanueva, G. R. 2002. Polliniferous plants and foraging strategies of Apis mellifera (Hymenoptera: Apidae) in the Yucatán Peninsula, Mexico. Revista de Biología Tropical, 50: 1035-1043.

Villanueva-Gutiérrez, R., Y. Moguel-Ordóñez, C. Echazarreta-González \& G. Arana-López. 2009. Monofloralhoneys in theYucatan Peninsula, Mexico. Grana, 48: 214-223.

Viuda-Martos, M., Y. Ruiz-Navajas, J. M. Zaldívar-Cruz, V. Kuri, J. Fernández-López, A. A. Carbonell-Barrachina \& J. A. Pérez-Álvarez. 2010. Aroma profile and physico-chemical properties of artisanal honey from Tabasco, Mexico. International Journal of Food Science and Techno$\log y, 45:$ 1111-1118. 